\title{
Treatment for metastatic adenocarcinoma of the stomach and gastroesophageal junction: 2020
}

\author{
Andrew Hsu ${ }^{1}$, Adam S. Zayac ${ }^{1}$, Aditya Eturi ${ }^{2}$, Khaldoun Almhanna ${ }^{1}$ \\ ${ }^{1}$ Division of Hematology/Oncology, The Warren Alpert Medical School of Brown University, Lifespan Cancer Institute, Rhode Island Hospital, \\ Providence, RI, USA; ${ }^{2}$ Department of Medicine, The Warren Alpert Medical School of Brown University, Providence, RI, USA \\ Contributions: (I) Conception and design: All authors; (II) Administrative support: None; (III) Provision of study materials or patients: None; (IV) \\ Collection and assembly of data: None; (V) Data analysis and interpretation: None; (VI) Manuscript writing: All authors; (VII) Final approval of \\ manuscript: All authors. \\ Correspondence to: Khaldoun Almhanna, MD. Division of Hematology/Oncology, The Warren Alpert Medical School of Brown University, Lifespan \\ Cancer Institute, Rhode Island Hospital, 593 Eddy St, George 353, Providence, RI 02903, USA. Email: Kalmhanna@lifespan.org.
}

\begin{abstract}
Gastric and gastroesophageal junction (GEJ) cancer is one of the most common malignancy worldwide. In unresectable or metastatic disease, the prognosis is poor and is generally less than a year. Standard front-line chemotherapy includes two- or three-drug regimens with the addition of trastuzumab in HER2-positive disease. With an increased understanding of the biology of cancer over the past few decades, targeted therapies have made their way into the treatment paradigm of many cancers. They been examined in the first- and second-line settings in the treatment of gastroesophageal cancer though has yielded few viable treatment options. One success is ramucirumab either as monotherapy or in combination with paclitaxel is the preferred choice in second-line therapy. While immunotherapy has been considered a breakthrough in oncology over the past decade, the response rates in gastric and gastroesophageal cancers have been relatively low compared to other cancers, resulting in its limited approval and mostly reserved for secondline therapy or beyond. In this article, we will review the standard first- and second-line treatment regimens. Furthermore, this article will review the use of targeted therapies and immunotherapy in treatment of gastric and gastroesophageal cancers. Lastly, we will touch upon future treatment strategies that are currently under investigation.
\end{abstract}

Keywords: Gastric cancer; gastroesophageal junction cancer; targeted therapy; immunotherapy; cytotoxic chemotherapy

Submitted Feb 01, 2020. Accepted for publication May 13, 2020.

doi: $10.21037 /$ atm-20-1159

View this article at: http://dx.doi.org/10.21037/atm-20-1159

\section{Introduction}

Gastric and gastroesophageal junction (GEJ) adenocarcinoma is the fifth most common cancer worldwide and the third leading cause of cancer-related mortality worldwide (1). The incidence of gastric adenocarcinoma varies depending on the region-with a high incidence in Asia, Latin America, Central and Eastern Europe, and low incidence in Western Europe and North America-that can be attributed to a decreased prevalence of Helicobacter pylori infections (2). In the United States, gastric adenocarcinoma is the fifteenth most common cancer, accounting for $1.6 \%$ of all new cancer diagnoses and $1.8 \%$ of cancer-related deaths in 2019 (3). Furthermore, the incidence of new cases and gastric cancer-related deaths have declined by $1.5 \%$ and $2.1 \%$ annually over the past decade, respectively (3). Despite the decreasing incidence of gastric adenocarcinoma in Western Europe and the United States, there has been an increasing incidence of GEJ adenocarcinoma (4).

Most patients with early-stage GEJ and gastric cancers are asymptomatic, though common presenting symptoms include anorexia, dyspepsia, weight loss, abdominal pain, 
and dysphagia in the cases where the tumor is located at the GEJ or proximal stomach (5). Given this insidious nature, nearly $40 \%$ of patients present with unresectable or metastatic disease at the time of diagnosis and the opportunity for cure through surgical resection is lost (3).

In metastatic disease, the prognosis is dismal and standard of care therapies have limited impact on patient outcomes-median survival ranges from four months with best supportive care (BSC) only, to 12 months with chemotherapy $(6,7)$. Systemic treatments for metastatic GEJ and gastric cancer consist of combination cytotoxic chemotherapy. Furthermore, targeted therapy with trastuzumab and ramucirumab has been incorporated in combination with cytotoxic chemotherapy in front-line and second-line treatment of GEJ and gastric adenocarcinoma respectively $(8,9)$. Lastly, the discovery of immune checkpoint inhibition has been considered a major medical and scientific breakthrough in the treatment of cancer; however, trials examining the use of immunotherapy either as monotherapy or in combination with cytotoxic chemotherapy in gastric and GEJ cancers have only led to limited approval in the second-line setting (after failure of initial therapy) with relatively low response rates ranging from $5-30 \%(10,11)$. The aim of this review article is to summarize the currently studied and approved treatments for GEJ and gastric cancer, as well as highlight some future treatments currently under investigation.

\section{Standard first-line therapy}

Several cytotoxic chemotherapeutics have shown activity against GEJ and gastric adenocarcinomasfluoropyrimidines (fluorouracil, capecitabine, S-1); platinums (cisplatin, oxaliplatin); taxanes (paclitaxel, docetaxel); anthracyclines (doxorubicin, epirubicin); and topoisomerase inhibitors (irinotecan). While these agents show activity when used as monotherapy, their objective response rates (ORR) are poor-fluoropyrimidines having an ORR 20-40\% (7,12), taxanes ORR 20\% (13), and irinotecan ORR 20\% (14). Furthermore, a 2010 Cochrane review showed an improvement in median overall survival (mOS) of 8.3 vs. 6.7 months with multi-agent cytotoxic chemotherapy compared single-agent fluorouracil (7). As a result, standard of care therapies for metastatic, HER-2 negative, GEJ and gastric adenocarcinoma consists of either two- or three-drug regimens for patients who are deemed fit for multi-agent chemotherapy.

The first "standard" regimen was established in 1980 and consisted of fluorouracil, doxorubicin, and mitomycin (FAM); this resulted in a partial response (PR) rate of $42 \%$ with a mOS was 5.5 months (15). In 1991, a new standard regimen was established after a randomized phase III trial compared FAM to fluorouracil, doxorubicin, and methotrexate (FAMTX). FAMTX demonstrated an ORR of $41 \%$ with $6 \%$ demonstrating a complete response compared to an ORR of $9 \%$ with $0 \%$ demonstrating a complete response with FAM $(\mathrm{P}<0.001)$. Furthermore, FAMTX demonstrated an improved mOS (9.7 vs. 6.7 months; $\mathrm{P}<0.004)$ (16). FAMTX remained the standard first-line regimen until 1997 when it was surpassed by epirubicin, cisplatin, and fluorouracil (ECF) which demonstrated improved ORR, $45 \%$ compared with $21 \%$ for FAMTX $(\mathrm{P}=0.0002)$ and improved mOS (8.9 vs. 5.7 months; $\mathrm{P}=0.0009)$ (17). These results were controversial as the mOS of FAMTX was worse than the previous studies, leading to questions over the true efficacy of epirubicin in addition to its added toxicities when compared to other regimens.

Next, the V325 study group examined the use of docetaxel, cisplatin and fluorouracil (DCF) versus cisplatin with fluorouracil (CF) and demonstrated improved mOS with DCF (9.2 vs. 8.6 months; $\mathrm{P}=0.02)$, improved ORR ( $37 \%$ vs. $25 \% ; \mathrm{P}=0.01$ ), and improved time to progression (TTP) (5.6 vs. 3.7 months; $\mathrm{P}<0.001$ ) (18). As expected, grade 3 or 4 toxicity with the triplet regimen was greater than with the doublet regimen-the most common being neutropenia (29\% vs. 12\%) (18). A subsequent phase III study examined the use of DCF with granulocyte colony-stimulating factor (gCSF) support versus modified DCF (mDCF) which consisted of a shorter duration of continuous infusion of fluorouracil along with decreased amounts of docetaxel and cisplatin. This modified regimen demonstrated improved grade 3 or 4 toxicity $-54 \%$ within the first 3 months (22\% hospitalized) and $76 \%$ over the course of treatment in the $\mathrm{mDCF}$ arm versus $71 \%$ toxicity within the first 3 months (52\% hospitalized) and $90 \%$ over the course of treatment in the DCF arm (19). The higher rate of grade 3 or 4 toxicity resulted in the DCF arm being closed prematurely in the study. Furthermore, mDCF demonstrated improved mOS when compared to DCF (18.8 vs. 12.6 months, respectively; $\mathrm{P}=0.007$ ) (19). Table 1 summarizes the landmark trials for first-line treatment of GEJ and gastric cancer.

The randomized phase III, REAL2 trial used a two-bytwo design to evaluate epirubicin-based triplet regimensepirubicin, cisplatin, fluorouracil (ECF); epirubicin, cisplatin, capecitabine (ECX); epirubicin, oxaliplatin, 
Table 1 Landmark trials in first-line treatment of GEJ and gastric cancers

\begin{tabular}{|c|c|c|c|c|c|}
\hline $\begin{array}{l}\text { Authors, } \\
\text { years (study name) }\end{array}$ & Treatment regimen & $\begin{array}{c}\text { Total } \\
\text { patients }\end{array}$ & ORR/CR & $\begin{array}{l}\text { mPFS (months); } \\
\text { HR, P value }\end{array}$ & $\begin{array}{l}\text { mOS (months) } \\
\text { HR, P value }\end{array}$ \\
\hline $\begin{array}{l}\text { MacDonald et al., } \\
1980\end{array}$ & Fluorouracil, doxorubicin \& mitomycin (FAM) & 62 & $42 \% / N R$ & NR & 5.5 \\
\hline Wils et al. 1991 & $\begin{array}{l}\text { Fluorouracil, doxorubicin \& methotrexate } \\
\text { (FAMTX) vs. FAM }\end{array}$ & 160 & $\begin{array}{c}41 \% / 6 \% \\
9 \% / 0 \%\end{array}$ & NR & 9.7 vs. 6.7 \\
\hline $\begin{array}{l}\text { Van Cutsem et al., } \\
2006 \text { (V325) }\end{array}$ & $\begin{array}{l}\text { Cisplatin \& fluorouracil (CF) vs. docetaxel, } \\
\text { cisplatin, fluorouracil (DCF) }\end{array}$ & 270 & $\begin{array}{l}37 \% / 2 \% \\
25 \% / 1 \%\end{array}$ & $\begin{array}{l}\text { TTP: } 5.6 \text { vs. } 3.7 \\
\text { HR 1.47, } P<0.001\end{array}$ & $\begin{array}{c}8.2 \text { vs. 9.6; } \\
\text { HR 1.29, } P=0.02\end{array}$ \\
\hline $\begin{array}{l}\text { Cunningham et al., } \\
2008 \text { (REAL2) }\end{array}$ & $\begin{array}{l}\text { Epirubicin, cisplatin, \& fluorouracil (ECF) vs. } \\
\text { epirubicin, cisplatin \& capecitabine (ECX); } \\
\text { epirubicin, oxaliplatin \& fluorouracil (EOF) } \\
\text { epirubicin, oxaliplatin \& capecitabine (EOX) }\end{array}$ & 1,002 & $\begin{array}{l}41 \% / 4 \% \\
46 \% / 4 \% \\
42 \% / 3 \% \\
48 \% / 4 \%\end{array}$ & $\begin{array}{l}6.2 \text { vs. } 6.7 \text { vs. } \\
6.5 \text { vs. } 7.0^{\dagger}\end{array}$ & $\begin{array}{l}9.9 \text { vs. } 9.9 \text { vs. } \\
9.3 \text { vs. } 11.2^{\dagger}\end{array}$ \\
\hline $\begin{array}{l}\text { Bang et al., } \\
2010^{\S} \text { (ToGA) }\end{array}$ & $\begin{array}{l}\text { Fluoropyrimidine, cisplatin \& trastuzumab } \\
\text { vs. fluoropyrimidine, cisplatin \& placebo }\end{array}$ & 594 & $\begin{array}{l}47 \% / 5 \% \\
35 \% / 2 \%\end{array}$ & $\begin{array}{l}6.7 \text { vs. } 5.5 ; \mathrm{HR} \\
0.71, \mathrm{P}=0.0002\end{array}$ & $\begin{array}{c}13.8 \text { vs. } 11.1 \\
\text { HR } 0.74, P=0.0046\end{array}$ \\
\hline
\end{tabular}

${ }^{\dagger}$, non-inferior; ${ }^{\ddagger}$, non-significant; ${ }^{\S}$, HER2-positive only. NR, not reported.

fluorouracil (EOF); and epirubicin, oxaliplatin, capecitabine (EOX). Based upon the predefined non-inferiority margin of 1.23, the four regimens (ECF, ECX, EOF, EOX) were non-inferior in terms of ORR ( $41 \%$ vs. $47 \%$ vs. $42 \%$ vs. $48 \%$, respectively), progression free survival (PFS) $(6.2$ vs. 6.7 vs. 6.5 vs. 7.0 months, respectively), and mOS (9.9 vs. 9.9 vs. 9.3 vs. 11.2 months, respectively) (20). For the capecitabine-fluorouracil (fluoropyrimidine) comparison, the hazard ratio (HR) was 0.86 (95\% CI, 0.80-0.90, noninferiority margin 1.23), and for the cisplatin-oxaliplatin (platinum) comparison, HR was 0.92 (95\% CI, 0.80-1.10, non-inferiority margin 1.23) (20). This suggested that that cisplatin could be substituted by oxaliplatin and fluorouracil could be substituted by capecitabine.

In Asia, S-1 is an oral fluoropyrimidine approved for the treatment of metastatic GEJ and gastric cancer. S-1 contains tegafur (a prodrug of fluorouracil), gimercil (a dihydropyrimidine dehydrogenase inhibitor, prolonging the half-life of fluorouracil), and oteracil potassium (an inhibitor of phosphorylation of intestinal fluorouracil, increasing gastrointestinal tolerability). In the SPIRITS trial, cisplatin plus S-1 (CS) was compared to S-1 monotherapy and demonstrated improved mOS with CS (13.0 vs. 11 months; $\mathrm{P}=0.04)$ and improved mPFS (6.0 vs. 4.0 months; $\mathrm{P}<0.0001)(21)$. In the subsequent
FLAGS trial, CS was compared to CF and did not find significant improvement in mOS (8.6 vs. 7.9 months, respectively; $\mathrm{P}=0.2$ ) (22). The phase III study, JCOG1013, added docetaxel to CS (DCS) and compared this to CS. Ultimately, no improvement in mOS was found (14.2 vs. 15.3 months, respectively; $\mathrm{P}=0.47$ ) (23). Regimens that include S-1 have been incorporated in the first-line treatment of metastatic GEJ and gastric cancer in Asia only.

Despite irinotecan demonstrating activity against metastatic GEJ and gastric adenocarcinoma when used as monotherapy, its incorporation into multi-agent regimens in front-line treatment has not been as successful (14). A subsequent phase III trial comparing irinotecan with fluorouracil (IF) versus CF failed to demonstrate superiority in mOS (9.0 vs. 8.7 months, respectively; $\mathrm{P}=0.53$ ) or TTP (5.0 vs. 4.2 months, respectively; $\mathrm{P}=0.088$ ) (24). Other phase III trials incorporating the use of irinotecan in the treatment of metastatic GEJ and gastric adenocarcinoma have failed to show superiority of irinotecan-based regimens over platinum-based regimens $(25,26)$. As a result, irinotecan should be considered when platinum-based chemotherapy cannot be tolerated in the first-line setting.

Several targeted agents have been studied for use in the first-line treatment in metastatic GEJ or gastric cancers. 
The results of these studies have been disappointing with only the HER2 antagonist, trastuzumab, currently approved for use in first-line treatment. A subset of GEJ and gastric adenocarcinomas exhibit HER2 amplification, resulting in HER2-receptor overexpression akin to some breast cancers. This has provided a site for targeted therapy using trastuzumab-approved in 2010 for first-line use in combination with chemotherapy in HER2-positive, advanced GEJ or gastric adenocarcinoma based on the ToGA trial (9). This phase III trial enrolling 584 patients compared a fluoropyrimidine with cisplatin plus trastuzumab versus a fluoropyrimidine with cisplatin only. Patients were required to have HER2 positivity, defined as either a score 3+ by immunohistochemistry or HER2:CEP17 ratio greater than or equal than 2 on FISH testing. The addition of trastuzumab provided both an improvement in mOS (13.8 vs. 11.1 months, respectively; HR 0.74, 95\% CI, 0.60-0.91; $\mathrm{P}=0.0046)$ and mPFS (6.7 vs. 5.5 months, respectively; HR 0.71; 95\% CI, 0.59-0.85; $\mathrm{P}=0.0002)$. Furthermore, the addition of trastuzumab to chemotherapy provided an improved duration of response (6.9 vs. 4.8 months, respectively; HR $0.53,95 \% \mathrm{CI}, 0.40-0.73 ; \mathrm{P}<0.0001)$ and ORR ( $47 \%$ vs. $35 \%$, respectively; $\mathrm{P}=0.0017$ ) with prolonged TTP (7.1 vs. 5.6 months, respectively; HR 0.70, 95\% CI, $0.58-0.85 ; \mathrm{P}=0.0003$ ) (9). Overall rates of adverse events were not different with the addition of trastuzumab to chemotherapy, whether considering all grades or only grade 3-4 events; although patients who received trastuzumab had higher rates of grade 3-4 diarrhea (9). Subsequently, the LOGiC/TRIO-013 trial studied the use of the dual HER2 and endothelial growth factor receptor (EGFR) antagonist, lapatinib, with capecitabine and oxaliplatin and found no improvement in progression free or overall survival (27).

Bevacizumab, a monoclonal antibody against vascular endothelial growth factor (VEGF), was evaluated for first-line use in the AVAGAST trial. In this phase III trial, patients were randomized to bevacizumab plus fluoropyrimidine-cisplatin or placebo plus fluoropyrimidine-cisplatin and found a non-significant improvement in mOS (12.1 vs. 10.1 months, respectively; $\mathrm{P}=0.1002)$; however, there was a significant improvement in mPFS (6.7 vs. 5.3 months, respectively; $\mathrm{P}=0.0037)$ (28). Ramucirumab, another VEGF antagonist, was recently examined in the first-line setting in the RAINFALL trial. Patients with metastatic, HER2-negative, GEJ or gastric adenocarcinoma were assigned to receive ramucirumab plus a fluoropyrimidine and cisplatin or placebo plus a fluoropyrimidine and cisplatin. This phase III trial failed to demonstrate significant improvement in mOS (11.7 vs. 10.7 months, respectively; $\mathrm{P}=0.6757)$, but showed marginal improvement in mPFS (5.7 vs. 5.4 months, respectively; $\mathrm{P}=0.0106$ ) (29). As a result, VEGF antagonists do not currently have a role in the first-line treatment of metastatic, HER2-negative, GEJ or gastric adenocarcinoma.

Further studies evaluating the agents targeting EGFR, cetuximab (EXPAND) and panitumumab (REAL3), with chemotherapy failed to show improvement in survival (30,31). In addition, the MET inhibitors onartuzumab (METGastric) and rilotumumab (RILOMET-1) also did not improve survival when added to first-line chemotherapy $(32,33)$. The hedgehog pathway inhibitor, vismodegib, also failed to improve either PFS or OS when added to fluorouracil and oxaliplatin in the first-line setting (34).

CLDN18.2 is present in approximately $50-70 \%$ of GEJ and gastric cancers and has led to the development of the monoclonal antibody, zolbetuximab (35). In the phase II FAST trial, zolbetuximab plus EOX was compared to EOX alone for first-line use in patients with advanced, HER-2 negative, CLDN18.2 expression (defined as $\geq 2+$ staining with anti-CLDN18 antibodies). Zolbetuximab with EOX demonstrated improved mPFS (7.5 vs. 5.3 months; HR 0.44, 95\% CI, 0.29-0.67; $\mathrm{P}<0.0005)$, improved mOS (13.0 vs. 8.4 months; HR $0.56,95 \% \mathrm{CI}, 0.40-0.67 ; \mathrm{P}=0.0008)$, and higher ORR (39\% vs. $25 \%$; $\mathrm{P}=0.022$ ) (36). Based upon this promising data, the larger phase III SPOTLIGHT trial is ongoing and examining the first-line use of zolbetuximab with fluorouracil and oxaliplatin versus fluorouracil and oxaliplatin alone (37).

Currently in the United States, the most common firstline regimen for metastatic, HER2-negative, GEJ and gastric adenocarcinoma consists of a fluoropyrimidine with a platinum $(18,20)$. Docetaxel may be added to form a triplet regimen in patients with good performance status and organ function $(18,19)$. For metastatic, HER2-positive disease, the first-line regimen consists of a fluoropyrimidine-platinum doublet plus trastuzumab (9).

\section{Standard second-line therapy}

In the second-line setting, the impact of cytotoxic chemotherapy on OS is very modest. A phase III study examined the use of irinotecan versus BSC in the secondline setting and found an improvement in mOS (4.0 vs. 2.4 months, respectively; HR 0.48, 95\% CI, 0.25-0.92; $\mathrm{P}=0.012$ ) (38). Another phase III study compared salvage chemotherapy with either docetaxel or irinotecan against 
Table 2 Landmark trials in second-line treatment of GEJ and gastric cancers

\begin{tabular}{|c|c|c|c|c|c|}
\hline $\begin{array}{l}\text { Authors, years } \\
\text { (study name) }\end{array}$ & Treatment regimen & $\begin{array}{c}\text { Total } \\
\text { patients }\end{array}$ & ORR/CR & $\begin{array}{l}\text { mPFS (months) } \\
\text { HR, P value }\end{array}$ & $\begin{array}{l}\text { mOS (months); } \\
\text { HR, P value }\end{array}$ \\
\hline $\begin{array}{l}\text { Fuchs et al., } 2014 \\
\text { (REGARD) }\end{array}$ & $\begin{array}{l}\text { Ramucirumab \& best supportive care } \\
\text { vs. placebo \& best supportive care }\end{array}$ & 355 & $\begin{array}{c}3 \% /<1 \% \\
3 \% / 0 \%\end{array}$ & $\begin{array}{c}2.1 \text { vs. } 1.3 \\
\text { HR } 0.483, P<0.0001\end{array}$ & $\begin{array}{c}5.2 \text { vs. } 3.8 ; \\
\text { HR } 0.776, P=0.47\end{array}$ \\
\hline $\begin{array}{l}\text { Wilke et al., } 2019 \\
\text { (RAINBOW) }\end{array}$ & $\begin{array}{l}\text { Ramucirumab \& paclitaxel vs. } \\
\text { placebo \& paclitaxel }\end{array}$ & 665 & $\begin{array}{l}28 \% /<1 \% \\
16 \% /<1 \%\end{array}$ & $\begin{array}{c}4.4 \text { vs. } 2.9 \\
\text { HR } 0.635, P<0.0001\end{array}$ & $\begin{array}{c}9.6 \text { vs. } 7.4 ; \\
\text { HR } 0.807, \mathrm{P}=0.017\end{array}$ \\
\hline $\begin{array}{l}\text { Kang et al., } 2017 \\
\text { (ATTRACTION-2) }\end{array}$ & Nivolumab vs. placebo & 493 & $\begin{array}{c}11.2 \% / 0 \% \\
0 \% / 0 \%\end{array}$ & $\begin{array}{c}1.61 \text { vs. } 1.45 \\
\text { HR } 0.60, P<0.0001\end{array}$ & $\begin{array}{c}5.26 \text { vs. } 4.14 \\
\text { HR } 0.63, \mathrm{P}<0.0001\end{array}$ \\
\hline $\begin{array}{l}\text { Doi et al., } 2019 \\
\text { (JAVELIN) }\end{array}$ & Avelumab & 40 & $10 \% / 2.5 \%$ & 2.4 & 9.1 \\
\hline
\end{tabular}

NR, not reported.

BSC in the second-line setting and demonstrated an improvement in mOS (5.3 vs. 3.8 months, respectively; HR 0.657, 95\% CI, 0.485-0.891; $\mathrm{P}=0.007$ ) (39). COUGAR-02, an open-labeled phase III study, examined the use of docetaxel versus BSC in the second-line setting in patients with esophageal, GEJ, or gastric adenocarcinoma that had progressed on or within 6 months of treatment with a fluoropyrimidine-platinum regimen and demonstrated an improved mOS (5.2 vs. 3.6 months, respectively; HR 0.67, 95\% CI, 0.49-0.92; $\mathrm{P}=0.01$ ) (40). In the ABSOLUTE trial, nanoparticle albumin-bound paclitaxel (nab-paclitaxel), administered either weekly or every three weeks, was compared to solvent-bound paclitaxel, administered weekly. This phase III trial demonstrated non-inferiority between the weekly nab-paclitaxel and solvent-bound paclitaxel regimens in terms of mOS, $11.1 v s .10 .9$ months, respectively (HR 0.97, 97.5\% CI, 0.76-1.23; non-inferiority margin 1.25; one-sided $\mathrm{P}=0.0085$ ); however, the every three week nab-paclitaxel regimen did not meet the noninferiority threshold for mOS, 10.3 vs. 10.9 months (HR $1.06,97.5 \%$ CI, 0.87-1.31; one-sided $\mathrm{P}=0.062$ ) (41). Given the marginal improvements in OS with cytotoxic chemotherapy alone, there have been numerous studies incorporating either targeted therapy or immunotherapy in the second-line setting.

Despite the failure of anti-VEGF agents to show significant improvement in OS in the front-line setting, ramucirumab was studied in second-line setting. In the phase III REGARD trial, patients who had progression after first-line platinum-containing, or fluoropyrimidinecontaining, chemotherapy were randomized to receive ramucirumab with BSC or placebo with BSC. The ramucirumab arm demonstrated improvement in $\mathrm{mOS}$ (5.2 vs. 3.8 months; HR 0.776, 95\% CI, 0.603-0.998; $\mathrm{P}=0.047$ ) leading to its initial approval as monotherapy in the second-line setting for GEJ or gastric adenocarcinoma in 2014 (42). The subsequent RAINBOW trial examined patients who had progressed on, or within 4 months, of first-line chemotherapy (fluoropyrimidine-platinum with or without an anthracycline) and were assigned to receive ramucirumab plus paclitaxel or placebo plus paclitaxel. Median OS was significantly improved in the ramucirumab plus paclitaxel (9.6 vs. 7.4 months; HR $0.807,95 \%$ CI, 0.678-0.962; $\mathrm{P}=0.0169$ ) (8). As a result of the outcomes from the RAINBOW study, ramucirumab plus paclitaxel is now the preferred choice for second-line therapy. Currently, there are ongoing trials examining the use of ramucirumab with irinotecan (RINDBeRG trial) and with fluorouracil, leucovorin, irinotecan (FOLFIRIram) $(43,44)$. Table 2 summarizes select landmark trials for second-line treatment of GEJ and gastric cancer.

The use of targeted agents in the second-line setting has yielded few viable treatment options. The phase II/ III GATSBY study investigated the efficacy of secondline trastuzumab emtansine (T-DM1) compared with second-line taxane therapy, but failed to improve overall survival (45). Of note, this study included nearly $80 \%$ of patients who had received prior HER2-directed therapy (45). Lapatinib was studied in the phase III TyTAN trial in combination with paclitaxel. While the addition of lapatinib to paclitaxel improved ORR (OR 3.85, $\mathrm{P}<0.001)$ compared with paclitaxel alone, it 
failed to improve mPFS or mOS (46). The use of the mTOR inhibitor, everolimus, was studied alone and in combination with paclitaxel after progression on first-line treatment in the phase III GRANITE-1 and RADPAC studies, respectively. Everolimus, whether as monotherapy, or in combination with paclitaxel, failed to improve PFS or OS $(47,48)$. The phase II Shine study assessed the fibroblast-growth factor receptor (FGFR) 1-3 inhibitor, AZD4547, compared with paclitaxel in patients with FGFR2 gene amplification or polysomy. Despite promising phase I study data, no improvement in PFS was observed (49). The combination of the oral poly (ADP-ribose) polymerase (PARP) inhibitor, olaparib, with paclitaxel failed to improve survival compared with paclitaxel alone among Asian patients with advanced gastric cancer who progressed despite first-line therapy in the phase III GOLD study (50).

Trifluridine (FTD) is a thymidine analogue that is combined with the thymidine phosphorylase inhibitor, tipiracil (TPI). The TAS-102 Gastric Study (TAGS) examined 507 metastatic gastric and GEJ cancer patient that were randomized 2:1 to receive FTD/TPI or placebo plus best supportive care. In patients with a prior gastrectomy, this phase III trial demonstrated an improved mOS (6.0 vs. 3.4 months; HR $0.57,95 \%$ CI, 0.41-0.79), mPFS (2.2 vs. 1.8 months; HR 0.48, 95\% CI, 0.35-0.65). Furthermore, patients without a prior gastrectomy also demonstrated an improved mOS (5.6 vs. 3.8 months; HR 0.80, $95 \%$ CI, 0.60-1.06), mPFS (1.9 vs. 1.8 months; HR $0.65,95 \%$ CI, $0.49-0.85)(51)$.

\section{Immunotherapy in the treatment of gastric and gastroesophageal cancer}

In the last decade, immune checkpoint inhibitors (ICIs), have emerged on the treatment landscape for metastatic GEJ and gastric cancers with microsatellite instability (MSI) or deficient mismatch repair (dMMR). Furthermore, patients whose tumors demonstrate high microsatellite instability (MSI-H) have a better prognosis (52).

Pembrolizumab, a monoclonal antibody targeting programmed cell death protein-1 (PD-1), is the most studied ICI in GEJ and gastric cancers. It is the only immunotherapy approved for use in the secondline treatment of metastatic GEJ and gastric cancer with PD-L1 positive, MSI-H, or dMMR tumors and approved for use in the third-line setting for PDL1 positive disease (10). Based upon the findings of
KEYNOTE-059, pembrolizumab was FDA-approved in 2017 for the treatment of chemotherapy-refractory (defined as progression after two lines of therapy), PDL1-positive gastroesophageal cancers (Table 2). In this phase II study, 259 patients received pembrolizumab $200 \mathrm{mg}$ intravenously every three weeks until disease progression. The ORR was $11.6 \%$ with a $2.3 \%$ achieving a complete response. In patients with PD-L1 positive and PD-L1 negative tumors, the ORR was $15.5 \%$ and $6.4 \%$, respectively. Of note, ORR was $57 \%$ in MSI tumors compared to $9 \%$ in microsatellite stable (MSS) tumors (10). Recently, KEYNOTE-062 examined the use of pembrolizumab monotherapy versus pembrolizumab with cisplatin and fluorouracil/capecitabine versus cisplatin and fluorouracil/capecitabine for patients with newly diagnosed advanced or metastatic GEJ or gastric cancer whose tumors expressed PD-L1 [defined as Combined Positive Score (CPS) $\geq 1$ ]. In patients with PD-L1 expression, the addition of pembrolizumab to chemotherapy did not result in an improved mOS when compared to chemotherapy alone (12.5 vs. 11.1 months; HR $0.85,95 \%$ CI, $0.70-1.03)$ or mPFS (6.9 vs. 6.4 months; HR 0.84, 95\% CI, 0.70-1.02) (53). However, pembrolizumab monotherapy was non-inferior to chemotherapy alone in terms of mOS (10.6 vs. 11.1 months; HR 0.91, 95\% CI, 0.69-1.18; noninferiority margin 1.2$)$ despite a lower ORR (14.5\% vs. $36.8 \%)$. In patients whose tumors strongly expressed PD-L1 (CPS $\geq 10$ ), pembrolizumab monotherapy resulted in a significant improvement in mOS when compared to chemotherapy alone (17.4 vs. 10.8 months; HR 0.69, 95\% CI, 0.490.97) (53).

While KEYNOTE-059 led to pembrolizumab's FDAapproval in the second-line setting, there have been recent updates from the ongoing KEYNOTE trials-additional cohorts from KEYNOTE-059 evaluated pembrolizumab as monotherapy or in combination with chemotherapy (cisplatin, fluorouracil), in the first-line setting for advanced GEJ and gastric cancers (54-56). Patients receiving pembrolizumab monotherapy were required to have tumors who express PD-L1 (CPS $\geq 1$ ), while the combination therapy arm did not require this, though $64 \%$ of patients in the combination arm had tumors expressing PD-L1. The combination arm resulted in an ORR of $60 \%$, with $4 \%$ demonstrating a complete response, and mPFS of 6.6 months (54-56). In the pembrolizumab monotherapy arm, ORR was $25.8 \%$ with $6.5 \%$ achieving a complete response, and mPFS of 3.3 months (54-56). An ongoing 
phase II study examining the combination of oxaliplatin, capecitabine, trastuzumab, and pembrolizumab in HER2positive, GEJ or gastric adenocarcinoma regardless of PD-L1 expression has shown promise with an ORR 83\%, including $4 \%$ achieving a complete response, mPFS 11.4 months, and tolerable side effects (57). This promising preliminary data has led to the initiation of the phase III KEYNOTE-811 trial, along with several other ongoing phase II and III KEYNOTE trials scheduled for completion by March 2024.

Nivolumab, another PD-1 inhibitor, is approved for use as monotherapy in Asia, based upon the results of ATTRACTION-2. This phase III trial examined patients with unresectable, advanced or recurrent GEJ or gastric cancer refractory or intolerant to standard therapy, defined as two or more previous chemotherapy regimens and randomized them to receive nivolumab monotherapy or placebo with BSC. This trial was able to demonstrate improvement in mOS (5.26 vs. 4.14 months; HR $0.63,95 \%$ CI, 0.51-0.78; $\mathrm{P}<0.0001)$ along with improved mPFS (1.61 vs. 1.45 months; $\mathrm{P}<0.0001)(11)$. Nivolumab was also examined in combination with ipilimumab, an anti-cytotoxic T-lymphocyte-associated protein-4 (CTLA-4) antibody, in CheckMate-032. This phase I/II study examined patients with locally advanced or metastatic-chemotherapy refractory esophageal, GEJ, or gastric cancers regardless of PD-L1 or MSI status; however, investigators of CheckMate-032 did note that patients with PD-L1 positive and MSI-H tumors had more robust responses. These patients were randomized to receive nivolumab $3 \mathrm{mg} / \mathrm{kg}$ monotherapy (NIVO3), nivolumab $1 \mathrm{mg} / \mathrm{kg}$ plus ipilimumab $3 \mathrm{mg} / \mathrm{kg}$ (NIVO1 + IPI3), and nivolumab $3 \mathrm{mg} / \mathrm{kg}$ plus ipilimumab $1 \mathrm{mg} / \mathrm{kg}$ (NIVO3 + IPI1). This study was able to demonstrate ORR $12 \%$ vs. $24 \%$ vs. $8 \%$, respectively; mPFS $1.4 v s$. 1.4 vs. 1.6 months, respectively; and mOS 6.2 vs. 6.9 vs. 4.8 months, respectively (58). The higher response rate in the NIVO1 + IPI3 arm came at the cost of nearly double the toxicity rates and a seven-fold increase in treatment discontinuation when compared to the NIVO3 arm (58). Results of CheckMate-032 have prompted larger phase II and III studies in the United States investigating nivolumab's use in the first-line setting in combination with chemotherapy, other ICIs, or targeted therapy.

Avelumab, another PD-L1 inhibitor, is approved as monotherapy for unresectable or metastatic gastric cancer in Europe based upon the JAVELIN study. This phase $\mathrm{Ib}$ study sought to examine the use of avelumab in advanced
GEJ or gastric adenocarcinoma who progressed after one or two lines of fluoropyrimidine-platinum chemotherapy. Avelumab had modest results-ORR $10 \%$ in all groups, ORR $27.3 \%$ in tumors with PD-L1 expression (CPS $\geq 1$ ), mOS 9.1 months, but with low rates of grade 3 or 4 toxicity (7.5\%) (59). JAVELIN Gastric 300 is an ongoing phase III trial evaluating the use of avelumab versus chemotherapy (paclitaxel or irinotecan) in the third-line setting. Preliminary results have been disappointing and show that avelumab has not led to improvements in mOS (4.6 vs. 5.0 months; HR 1.1, 95\% CI, 0.9-1.4; $\mathrm{P}=0.81$ ) or mPFS (1.4 vs. 2.7 months; HR 1.73, 95\% CI, 1.4-2.2; P>0.99) (60). The phase III JAVELIN Gastric 100 trial evaluated avelumab as maintenance after first-line chemotherapy versus continuing first-line chemotherapy and demonstrated no improvement in mOS (10.4 vs. 10.9 months; HR 0.91, 95\% CI, 0.74-1.11; $\mathrm{P}=0.1779$ ) (61).

Despite the success of incorporating ICIs in the treatment of other solid tumors over the past decade, the gains in the treatment of GEJ and gastric cancer have been modest with relatively low response rates, although these are higher in patients with PD-L1 expressing, MSI-H, or dMMR disease. This has resulted in limited approval of immunotherapy, mostly reserved for use in second or subsequent lines of therapy-pembrolizumab (United States) (10), nivolumab (Asia) (11), and avelumab (Europe) (58). Currently, there are several ongoing trials examining the use of ICIs in combination with cytotoxic chemotherapy, targeted therapy, and other immunotherapies in the treatment of GEJ and gastric cancer.

\section{Conclusions}

Unfortunately, a large portion of patients with GEJ or gastric cancers are initially diagnosed with unresectable or metastatic disease given its insidious nature. Systemic treatments over the past four decades have led to modest improvement in overall survival when compared to BSC alone. In the front-line setting, these systemic treatments consist of a combination of a fluoropyrimidine and platinum with the addition of a docetaxel provided a patient has a good performance status and good organ function (18-22). Trastuzumab is added to a fluoropyrimidine and platinum doublet in patients with HER2-positive disease (9). In the second-line setting, ramucirumab with paclitaxel is the recommended regimen; however, in patients with PD-L1 expressing tumors, pembrolizumab is reasonable 
alternative option with a favorable side effect profile $(8,10)$. Given the modest gains in overall survival with the use of immunotherapy alone, there is a significant amount of ongoing research and trials examining the combination of immunotherapy with targeted therapy, cytotoxic chemotherapy, and other immune checkpoint inhibitors in hopes of further improving the overall survival for metastatic GEJ and gastric cancer.

\section{Acknowledgments}

Funding: None.

\section{Footnote}

Provenance and Peer Review: This article was commissioned by the Guest Editor (Dr. Khaldoun Almhanna) for the series "Gastroesophageal Cancer 2020" published in Annals of Translational Medicine. The article was sent for external peer review organized by the Guest Editor and the editorial office.

Conflicts of Interest: All authors have completed the ICMJE uniform disclosure form (available at http://dx.doi. org/10.21037/atm-20-1159). The series "Gastroesophageal Cancer 2020" was commissioned by the editorial office without any funding or sponsorship. KA served as the unpaid Guest Editor of the series and serves as an unpaid editorial board member of Annals of Translational Medicine from Nov 2019 to Oct 2021. Dr. Almhanna reports personal fees from Merck, outside the submitted work. The authors have no other conflicts of interest to declare.

Ethical Statement: The authors are accountable for all aspects of the work in ensuring that questions related to the accuracy or integrity of any part of the work are appropriately investigated and resolved.

Open Access Statement: This is an Open Access article distributed in accordance with the Creative Commons Attribution-NonCommercial-NoDerivs 4.0 International License (CC BY-NC-ND 4.0), which permits the noncommercial replication and distribution of the article with the strict proviso that no changes or edits are made and the original work is properly cited (including links to both the formal publication through the relevant DOI and the license). See: https://creativecommons.org/licenses/by-nc-nd/4.0/.

\section{References}

1. Fitzmaurice C, Allen C, Barber RM, et al. Global, Regional, and National Cancer Incidence, Mortality, Years of Life Lost, Years Lived With Disability, and DisabilityAdjusted Life-years for 32 Cancer Groups, 1990 to 2015: a Systematic Analysis for the Global Burden of Disease Study. JAMA Oncol 2017;3:524-48.

2. Ferro A, Peleteiro B, Malvezzi M, et al. Worldwide trends in gastric cancer mortality (1980-2011), with predictions to 2015, and incidence by subtype. Eur J Cancer 2014;50:1330-44.

3. SEER Cancer Stat Facts: Stomach Cancer Bethesda, MD: National Cancer Institute (NCI), 2019. Available online: https://seer.cancer.gov/statfacts/html/stomach.html

4. Pohl H, Sirovich B, Welch H. Esophageal adenocarcinoma incidence: are we reaching the peak? Cancer Epidemiol Biomarkers Prev 2010;19:1468-70.

5. Lutz MP, Zalcberg JR, Ducreux M, et al. Highlights of the EORTC St. Gallen international expert consensus on the primary therapy of gastric, gastroesophageal and oesophageal cancer-differential treatment strategies for subtypes of early gastroesophageal cancer. Eur J Cancer 2012;48:2941-53.

6. Glimelius B, Ekström K, Hoffman K, et al. Randomized comparison between chemotherapy plus best supportive care with best supportive care in advanced gastric cancer. Ann Oncol 1997;8:163-8.

7. Wagner AD, Unverzagt S, Grothe W, et al. Chemotherapy for advanced gastric cancer. Cochrane Database Syst Rev 2010;(3):CD004064.

8. Wilke H, Muro K, Van Cutsem E, et al. Ramucirumab plus paclitaxel versus placebo plus paclitaxel in patients with previously treated advanced gastric or gastro-oesophageal junction adenocarcinoma (RAINBOW): a double-blind, randomised phase 3 trial. Lancet Oncol 2014;15:1224-35.

9. Bang YJ, Van Cutsem E, Feyereislova A, et al. Trastuzumab in combination with chemotherapy versus chemotherapy alone for treatment of HER2-positive advanced gastric or gastro-oesophageal junction cancer (ToGA): a phase 3, open-label, randomised controlled trial. Lancet 2010;376:687-97.

10. Fuchs CS, Doi T, Jang RW, et al. Safety and efficacy of pembrolizumab monotherapy in patients with previously treated advanced gastric and gastroesophageal junction cancer: Phase 2 Clinical KEYNOTE-059 Trial. JAMA Oncol 2018;4:e180013. 
11. Kang YK, Boku N, Satoh T, et al. Nivolumab in patients with advanced gastric or gastro-oesophageal junction cancer refractory to, or intolerant of, at least two previous chemotherapy regimens (ONO-4538-12, ATTRACTION-2): a randomised, double-blind, placebocontrolled, phase 3 trial. Lancet 2017;390:2461-71.

12. Hong YS, Song SY, Lee SI, et al. A phase II trial of capecitabine in previously untreated patients with advanced and/or metastatic gastric cancer. Ann Oncol 2004;15:1344-7.

13. Sulkes A, Smyth J, Sessa C, et al. Docetaxel (Taxotere) in advanced gastric cancer:results of a phase II clinical trial. Br J Cancer 1994;70:380-83.

14. Köhne CH, Catane R, Klein B, et al. Irinotecan is active in chemonaive patients with metastatic gastric cancer: a phase II multicentric trial. Br J Cancer 2003;89:997-1001.

15. MacDonald JS, Schein PS, Woolley PV, et al. 5-fluorouracil, doxorubicin, and mitomycin (FAM) combination chemotherapy for advanced gastric cancer. Ann Intern Med 1980;93:533-36.

16. Wils JA, Klein HO, Wagener DJ, et al. Sequential highdose methotrexate and fluorouracil combined with doxorubicin-a step ahead in the treatment of advanced gastric cancer: a trial of the European Organization for Research and Treatment of Cancer Gastrointestinal Tract Cooperative Group. J Clin Oncol 1991;9:827-31.

17. Webb A, Cunningham D, Scarffe JH, et al. Randomized trial comparing epirubicin, cisplatin, and fluorouracil versus fluorouracil, doxorubicin, and methotrexate in advanced esophagogastric cancer. J Clin Oncol 1997;15:261-7.

18. Van Cutsem E, Moiseyenko V, Tjulandin S, et al. Phase III study of docetaxel and cisplatin plus fluorouracil compared with cisplatin and fluorouracil as first-line therapy for advanced gastric cancer: a report of the V325 Study Group. J Clin Oncol 2006;24:4991-7.

19. Shah MA, Janjigian YY, Stoller R, et al. Randomized Multicenter Phase II Study of Modified Docetaxel, Cisplatin, and Fluorouracil (DCF) Versus DCF Plus Growth Factor Support in Patients with Metastatic Gastric Adenocarcinoma: a Study of the US Gastric Cancer Consortium. J Clin Oncol 2015;33:3874-9.

20. Cunningham D, Starling N, Rao S, et al. Capecitabine and oxaliplatin for advanced esophagogastric cancer. N Engl J Med 2008;358:36-46.

21. Koizumi W, Narahara H, Hara T, et al. S-1 plus cisplatin versus $\mathrm{S}-1$ alone for first-line treatment of advanced gastric cancer (SPIRITS trial): a phase III trial. Lancet Oncol
2008;9:215-21.

22. Ajani JA, Rodriguez W, Bodoky G, et al. Multicenter phase III comparison of cisplatin/S-1 with cisplatin/infusional fluorouracil in advanced gastric or gastroesophageal adenocarcinoma study: the FLAGS trial. J Clin Oncol 2010;28:1547-53.

23. Yamada Y, Boku N, Mizusawa J, et al. Phase III study comparing triplet chemotherapy with $\mathrm{S}-1$ and cisplatin plus docetaxel versus doublet chemotherapy with S-1 and cisplatin for advanced gastric cancer (JCOG1013). J Clin Oncol 2018;36:abstr 4009.

24. Dank M, Zaluski J, Barone C, et al. Randomized phase III study comparing irinotecan combined with 5-fluorouracil and folinic acid to cisplatin combined with 5 -fluorouracil in chemotherapy naive patients with advanced adenocarcinoma of the stomach or esophagogastric junction. Ann Oncol 2008;19:1450-7.

25. Guimbaud R, Louvet C, Ries P, et al. Prospective, randomized, multicenter, phase III study of fluorouracil, leucovorin, and irinotecan versus epirubicin, cisplatin, and capecitabine in advanced gastric adenocarcinoma: a French intergroup (Fédération Francophone de Cancérologie Digestive, Fédération Nationale des Centres de Lutte Contre le Cancer, and Groupe Coopérateur Multidisciplinaire en Oncologie) study. J Clin Oncol 2014;32:3520-6.

26. Bouché O, Raoul JL, Bonnetain F, et al. Randomized multicenter phase II trial of a biweekly regimen of fluorouracil and leucovorin (LV5FU2), LV5FU2 plus cisplatin, or LV5FU2 plus irinotecan in patients with previously untreated metastatic gastric cancer: a Federation Francophone de Cancerologie Study-FFCD 9803. J Clin Oncol 2004;22:4319-28.

27. Hecht JR, Bang YJ, Qin SK, et al. Lapatinib in Combination With Capecitabine Plus Oxaliplatin in Human Epidermal Growth Factor Receptor 2-Positive Advanced or Metastatic Gastric, Esophageal, or Gastroesophageal Adenocarcinoma: tRIO-013/ LOGiC-A Randomized Phase III Trial. J Clin Oncol 2016;34:443-51.

28. Ohtsu A, Shah M, Van Cutsem E, et al. Bevacizumab in combination with chemotherapy as first-line therapy in advanced gastric cancer: a randomized, doubleblind, placebo-controlled phase III study. J Clin Oncol 2011;29:3968-76.

29. Fuchs CS, Shitara K, Bartolomeo MD, et al. RAINFALL: a randomized, double-blind, placebo-controlled phase III study of cisplatin (Cis) plus capecitabine (Cape) or 5FU 
with or without ramucirumab (RAM) as first-line therapy in patients with metastatic gastric or gastroesophageal junction (G-GEJ) adenocarcinoma. J Clin Oncol 2018;36:abstr 5 .

30. Lordick F, Kang YK, Chung HC, et al. Capecitabine and cisplatin with or without cetuximab for patients with previously untreated advanced gastric cancer (EXPAND): a randomised, open-label phase 3 trial. Lancet Oncol 2013;14:490-9.

31. Waddell T, Chau I, Cunningham D, et al. Epirubicin, oxaliplatin, and capecitabine with or without panitumumab for patients with previously untreated advanced oesophagogastric cancer (REAL3): a randomised, openlabel phase 3 trial. Lancet Oncol 2013;14:481-9.

32. Shah MA, Bang YJ, Lordick F, et al. Effect of Fluorouracil, Leucovorin, and Oxaliplatin With or Without Onartuzumab in HER2-Negative, MET-Positive Gastroesophageal Adenocarcinoma: the METGastric Randomized Clinical Trial. JAMA Oncol 2017;3:620-7.

33. Catenacci DVT, Tebbutt NC, Davidenko I, et al. Rilotumumab plus epirubicin, cisplatin, and capecitabine as first-line therapy in advanced MET-positive gastric or gastro-oesophageal junction cancer (RILOMET-1): a randomised, double-blind, placebo-controlled, phase 3 trial. Lancet Oncol 2017;18:1467-82.

34. Cohen DJ, Christos PJ, Kindler HL, et al. Vismodegib (V), a hedgehog $(\mathrm{HH})$ pathway inhibitor, combined with FOLFOX for first-line therapy of patients (pts) with advanced gastric and gastroesophageal junction (GEJ) carcinoma: a New York Cancer Consortium led phase II randomized study. J Clin Oncol 2013;31:4011.

35. Sahin U, Koslowski M, Dhaene Ket al. Claudin-18 splice variant 2 is a pan-cancer target suitable for therapeutic antibody development. Clin Cancer Res 2008;14:7624-34.

36. Sahin U, Tureci Ö, Manikhas GM, et al. Zolbetuximab combined with EOX as first-line therapy in advanced CLDN18.2 + gastric (G) and gastroesophageal junction (GEJ) adenocarcinoma:updated results from the FAST trial. J Clin Oncol 2019;37:16.

37. A Phase 3 Efficacy, Safety, and Tolerability Study of Zolbetuximab (Experimental Drug) Plus mFOLFOX6 Chemotherapy Compared to Placebo Plus mFOLFOX6 as Treatment for Gastric and Gastroesophageal Junction (GEJ) Cancer (SPOTLIGHT). Available online: https:// ClinicalTrials.gov/show/NCT03504397

38. Thuss-Patience PC, Kretzschmar A, Bichev D, et al. Survival advantage for irinotecan versus best supportive care as second-line chemotherapy in gastric cancer- a randomised phase III study of the Arbeitsgemeinschaft Internistische Onkologie (AIO). Eur J Cancer 2011;47:2306-14.

39. Kang JH, Lee SI, Lim DH, et al. Salvage chemotherapy for pretreated gastric cancer: a randomized phase III trial comparing chemotherapy plus best supportive care with best supportive care alone. J Clin Oncol 2012;30:1513-8.

40. Ford HE, Marshall A, Bridgewater JA, et al. Docetaxel versus active symptom control for refractory oesophagogastric adenocarcinoma (COUGAR-02): an open-label, phase 3 randomised controlled trial. Lancet Oncol 2014;15:78-86.

41. Shitara K, Takashima A, Fujitani K. Nab-paclitaxel versus solvent-based paclitaxel in patients with previously treated advanced gastric cancer (ABSOLUTE): an openlabel, randomised, non-inferiority, phase 3 trial. Lancet Gastroenterol Hepatol 2017;2:277-87.

42. Fuchs CS, Tomasek J, Yong CJ, et al. Ramucirumab monotherapy for previously treated advanced gastric or gastrooesophageal junction adenocarcinoma (REGARD): an international, randomised, multicentre, placebocontrolled, phase 3 trial. Lancet 2014;383:31-9.

43. Sakai D, Boku N, Kodera Y, et al. An intergroup phase III trial of ramucirumab plus irinotecan in third or more line beyond progression after ramucirumab for advanced gastric cancer (RINDBeRG trial). J Clin Oncol 2018. doi: 10.1200/JCO.2018.36.15_suppl.TPS4138.

44. Klempner SJ, Maron SB, Chase L, et al. Initial Report of Second-Line FOLFIRI in Combination with Ramucirumab in Advanced Gastroesophageal Adenocarcinomas: a Multi-Institutional Retrospective Analysis. Oncologist 2019;24:475-82.

45. Thuss-Patience PC, Shah MA, Ohtsu A, et al. Trastuzumab emtansine versus taxane use for previously treated HER2positive locally advanced or metastatic gastric or gastrooesophageal junction adenocarcinoma (GATSBY): an international randomised, open-label, adaptive, phase $2 / 3$ study. Lancet Oncol 2017;18:640-53.

46. Satoh T, Xu RH, Chung HC, et al. Lapatinib Plus Paclitaxel Versus Paclitaxel Alone in the Second-Line Treatment of HER2-Amplified Advanced Gastric Cancer in Asian Populations: tyTAN-A Randomized, Phase III Study. J Clin Oncol 2014;32:2039-49.

47. Ohtsu A, Ajani JA, Bai YX, et al. Everolimus for Previously Treated Advanced Gastric Cancer:Results of the Randomized, Double-Blind, Phase III GRANITE-1 Study. J Clin Oncol 2013;31:3935-43.

48. Al-Batran SE, Riera-Knorrenschild J, Pauligk C, et al. A 
randomized, double-blind, multicenter phase III study evaluating paclitaxel with and without RAD001 in patients with gastric cancer who have progressed after therapy with a fluoropyrimidine/platinum-containing regimen (RADPAC). J Clin Oncol 2017;35:4.

49. Van Cutsem E, Bang YJ, Mansoor W, et al. A randomized, open-label study of the efficacy and safety of AZD4547 monotherapy versus paclitaxel for the treatment of advanced gastric adenocarcinoma with FGFR2 polysomy or gene amplification. Ann Oncol 2017;28:1316-24.

50. Bang $\mathrm{YJ}, \mathrm{Xu} \mathrm{RH}$, Chin $\mathrm{K}$, et al. Olaparib in combination with paclitaxel in patients with advanced gastric cancer who have progressed following first-line therapy (GOLD): a double-blind, randomised, placebo-controlled, phase 3 trial. Lancet Oncol 2017;18:1637-51.

51. Ilson DH, Tabernero J, Prokharau A, et al. Efficacy and safety of trifluridine/tipiracil treatment in patients with metastatic gastric cancer who had undergone gastrectomy: subgroup analyses of a randomized clinical trial. JAMA Oncol 2019. doi:10.1001/jamaoncol.2019.3531.

52. Le DT, Durham JN, Smith KN, et al. Mismatch repair deficiency predicts response of solid tumors to PD-1 blockade. Science 2017;357:409-13.

53. Tabernero J, Cutsem EV, Bang YJ, et al. Pembrolizumab with or without chemotherapy versus chemotherapy for advanced gastric or gastroesophageal junction (G/GEJ) adenocarcinoma: the phase III KEYNOTE-062 study. J Clin Oncol 2019;37:LBA4007.

54. A Study of Pembrolizumab (MK-3475) in Participants With Recurrent or Metastatic Gastric or Gastroesophageal Junction Adenocarcinoma (MK-3475-059/ KEYNOTE-059). Available online: https://ClinicalTrials. gov/show/NCT02335411

55. Bang YJ, Kang YK, Catenacci DV, et al. Pembrolizumab alone or in combination with chemotherapy as firstline therapy for patients with advanced gastric or

Cite this article as: Hsu A, Zayac AS, Eturi A, Almhanna K. Treatment for metastatic adenocarcinoma of the stomach and gastroesophageal junction: 2020. Ann Transl Med 2020;8(17):1109. doi: 10.21037/atm-20-1159 gastroesophageal junction adenocarcinoma: results from the phase II nonrandomized KEYNOTE-059 study. Gastric Cancer 2019;22:828-37.

56. Wainberg ZA, Yoon HH, Catenacci DVT, et al. Efficacy and safety of pembrolizumab (pembro) alone or in combination with chemotherapy (chemo) in patients (pts) with advanced gastric or gastroesophageal (G/GEJ) cancer: Long-term follow up from KEYNOTE-059. J Clin Oncol 2019;37:4009.

57. Janjigian YY, Maron SB, Chou JF, et al. First-line pembrolizumab $(\mathrm{P})$, trastuzumab $(\mathrm{T})$, capecitabine (C) and oxaliplatin $(\mathrm{O})$ in HER2-positive metastatic esophagogastric adenocarcinoma. J Clin Oncol 2019;37:4011.

58. Janjigian YY, Ott PA, Calvo E, et al. Nivolumab \pm ipilimumab in pts with advanced (adv)/metastatic chemotherapy-refractory (CTx-R) gastric $(\mathrm{G})$, esophageal (E), or gastroesophageal junction (GEJ) cancer: CheckMate 032 study. J Clin Oncol 2017;35:4014.

59. Doi T, Iwasa $\mathrm{S}$, Muro K, et al. Phase 1 trial of avelumab (anti-PD-L1) in Japanese patients with advanced solid tumors, including dose expansion in patients with gastric or gastroesophageal junction cancer: the JAVELIN Solid Tumor JPN trial. Gastric Cancer 2019;22:817-27.

60. Bang YJ, Ruiz EY, Van Cutsem E, et al. Phase III, randomised trial of avelumab versus physician's choice of chemotherapy as third-line treatment of patients with advanced gastric or gastro-oesophageal junction cancer: primary analysis of JAVELIN Gastric 300. Ann Oncol 2018;29:2052-60.

61. Moehler MH, Dvorkin M, Ozguroglu M, et al. Results of the JAVELIN Gastric 100 phase 3 trial: avelumab maintenance following first-line (1L) chemotherapy (CTx) vs. continuation of $\mathrm{CT} x$ for HER2- advanced gastric or gastroesophageal junction cancer (GC/GEJC). J Clin Oncol 2020;38:278. 